Int. J. Dev. Biol. 49: 981-984 (2005)

doi: $10.1387 / \mathrm{ijdb} .052070 \mathrm{jl}$

Developmental Expression Pattern

\title{
Developmental expression of Xenopus Fragile $X$ mental retardation-1 gene
}

\author{
JAE H. LIM ${ }^{1}$, TING LUO ${ }^{2}$, THOMAS D. SARGENT ${ }^{2}$ and JUSTIN R. FALLON*,1 \\ ${ }^{1}$ Department of Neuroscience, Brown University, Providence, RI, USA and ${ }^{2}$ Laboratory of Molecular Genetics, NICHD, NIH, Bethesda, USA
}

\begin{abstract}
Dysregulation of Fragile X mental retardation-1 (Fmr1) gene expression results in an inherited form of mental retardation known as the Fragile $X$ syndrome (FXS). Fmr1 is a highly conserved gene with a broad yet distinctive expression pattern during vertebrate development. Here, we examined the expression pattern of Fmr1 during Xenopus embryonic development. Zygotic expression of Fmr1 began just prior to gastrulation and gradually increased during subsequent embryonic stages. By in situ hybridization, Fmr1 transcripts were detected by early tailbud stage and showed robust expression in the central nervous system (CNS), eye and pharyngeal arches. By late tailbud stage, Fmr1 expression became stronger in the CNS and craniofacial regions including the ear vesicle and eye. In addition, the notochord expressed high levels of Fmr1 transcripts in the late tailbud stage embryos. In the tadpole brain, the olfactory bulb and cerebellum exhibited strong Fmr1 expression. The developmental expression pattern of Fmr1 is consistent with the wide range of abnormalities observed in FXS. Further, our findings indicate that Xenopus will serve as an excellent model to study the developmental basis of this disease.
\end{abstract}

KEY WORDS: Fmr1, Fragile X syndrome, neural crest, Xenopus

Fragile $X$ syndrome (FXS) is the most common form of inherited mental retardation, with an incidence of about 1:4000 in males and 1:8000 in females. Individuals with FXS display moderate to severe mental retardation as well as craniofacial abnormalities and connective tissue dysplasia (Hagerman and Hagerman, 2002). Fragile $X$ mental retardation-1 (Fmr1) gene encodes an RNA-binding protein, FMRP, which is highly conserved during evolution. FMRP consists of two K-homology (KH1-2) domains and an RGG-box domain (Fig. 1A). It also has a nuclear localization (NLS) and an export (NES) signal (Fig. 1A). Recently, the RGG-box has been demonstrated to mediate binding to a subset of RNAs with G-quartet structure (Darnell et al., 2001). Xenopus FMRP had $90 \%, 95 \%, 90 \%, 100 \%$ and $61 \%$ amino acid sequence identities to NLS, KH1, KH2, NES and RGG box domains of human FMRP, respectively (Fig. 1A). Phylogenetic analysis further revealed that the amino acid sequences of Xenopus FMRP is closer to human FMRP than either zebrafish or Drosophila (Fig. 1B), suggesting that it will serve as a useful model to study FMRP function(s).

Northern blot analysis of developing embryos indicated that Xenopus Fmr1 has a large maternal component, most of which disappeared prior to gastrulation (Fig. 2). Zygotic expression of Fmr1 began at about gastrula (st. 10) and gradually increased from neurula (st. 12) to early tadpole stage (st. 47). During early to late tailbud stage, Fmr1 expression intensified in the craniofacial region with prominent staining in the pharyngeal arches (Fig. 3A). This distribution resembled the expression pattern of neural crest marker $A P$-2 $\alpha$ (compare Fig. 3A and 3B). However, unlike the $A P-2 \alpha$ expression at this stage (Luo et al., 2002), Fmr1transcript levels were higher in the CNS and eye (Fig. 3A, $E$ and $G$ ).

Horizontal sections of an early tailbud stage embryo further revealed that endodermal, mesodermal and mesenchymal (neural crest) regions of the pharyngeal arches expressed Fmr1 (Fig. 3A and D). By early tadpole stage (st. 35), forebrain, midbrain, hindbrain regions and the notochord showed high Fmrlexpression (Fig. 3C, F and G). Similar findings in the CNS (Agulhon et al., 1999) and notochord (Hergersberg et al., 1995) have been observed in mice. Again, craniofacial regions including the ear vesicles (Fig. 3F) and eye (Fig. 3G) displayed high Fmr1 transcript levels at this stage. Furthermore, in the late tadpole (st. 45) CNS, the olfactory bulbs and the cerebellum displayed strong Fmr1 expression (Fig. 4).

Abbreviations used in this paper: CNS, central nervous system; Fmr, Fragile X mental retardation; FXS, Fragile X syndrome

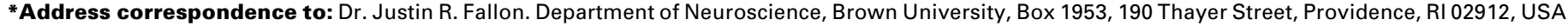
Fax: +1-401-863-1074. e-mail: Justin_Fallon@brown.edu
} 
A

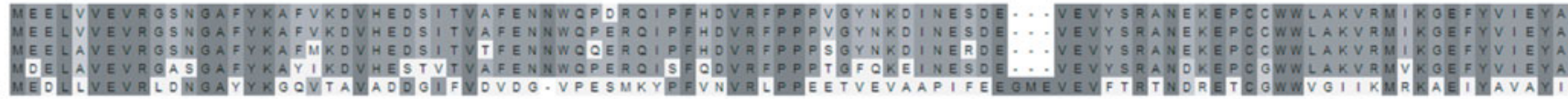

Mouse

Zebrafish

Human

Mouse

Xenopus

Zebrafish

Drosophila

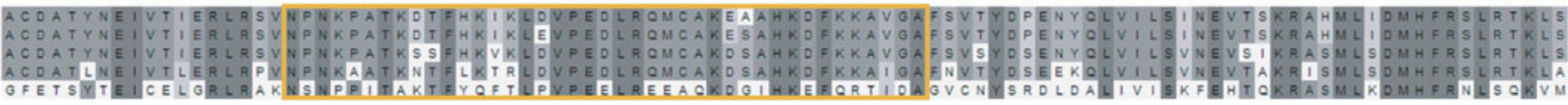

Human

Mouse

Xenopus

Drosophila

Human

Mouse

Xenopus

Drosophila
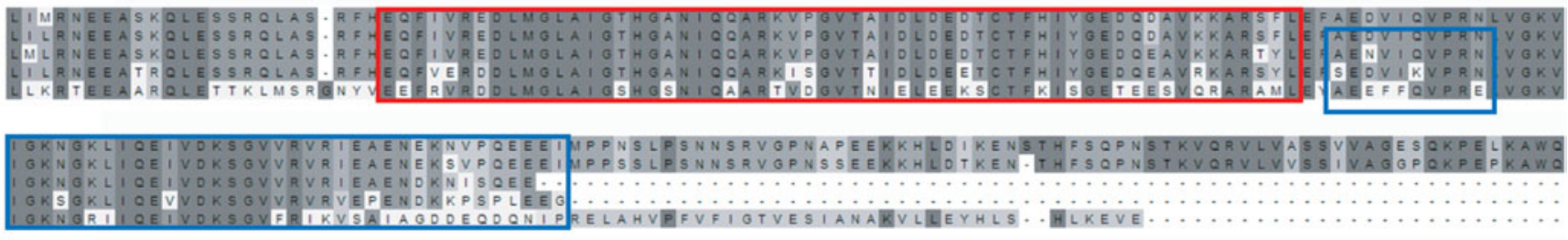

Human

Xense

Xenopus

Drosophila

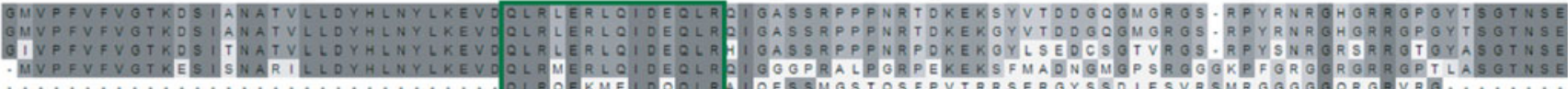

Human

Mouse

Xenopus

Zebrafish

Human ONTSSEOSRLRTOKDRNOKKEKPOSVDGOOPLVNOVP.

Mouse

Xenopus

AAMAOARGQNRORPIKERYMKKEKODOSDS OAYYYO

SOAMAOAROONRORPIKERVMKKEKODOSOSOAVVNOVS.

\section{Human \\ Mouse \\ Xenopus \\ Drosophila}

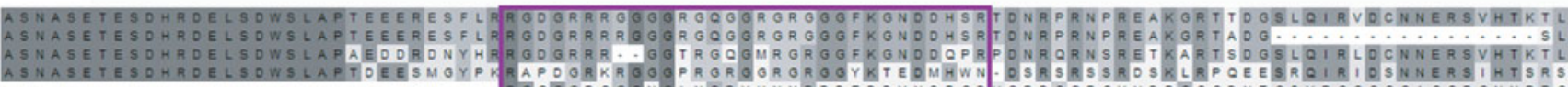

OSASSEOSRLRTOKORNOKKEKPOSVOOLOPLVNOVF.

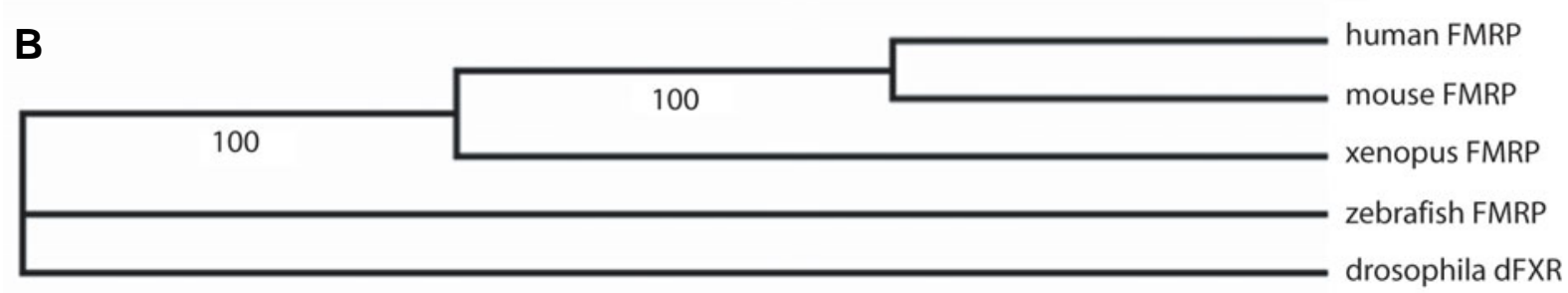

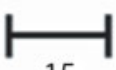

15
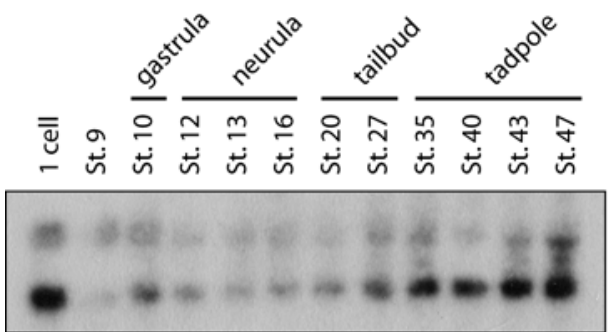

xFmr1

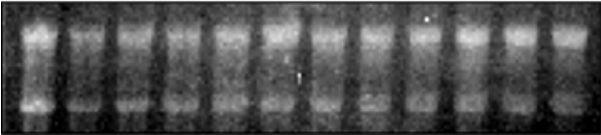

$28 \mathrm{~s}$

$18 \mathrm{~s}$
Fig. 1 (Above). ClustalW alignment of translated Fmr1 gene from human, mouse, Xenopus, zebrafish and Drosophila. (A) The common motifs of FMRP conserved in all analyzed species are highlighted by colored boxes lorange: NLS, nuclear localization signal; red: KH1, K Homology domain 1; blue: KH2, Khomology domain 2; green: NES, nuclear export signal; purple: RGG box). (B) Phylogenetic tree of FMRP sequences from above species constructed by DS Gene 1.5 program. The total number of differences between sequences is indicated by the scale bar and the bootstrap support values are shown at the node of the tree as a percentage. The amino acid sequences of human, mouse, Xenopus, zebrafish FMRP and Drosophila dFMR1 were obtained from GenBank database with accession numbers AAB18832, AAL66364, P51113, NP_694495 and AAF14639, respectively.

Fig. 2 (Left). Northern blot analysis of Fmr1 expression during Xenopus embryonic development. Fmr1 transcripts are approximately $2.1 \mathrm{~kb}$ (bottom band; top band is non-specific) and are present maternally (1 cell). Zygotic expression begins at early gastrula (st. 10) and gradually increases through tadpole stage (st. 47). A noticeable spike in Fmr1 trancript levels is observed from st. 20 to st. 27. 

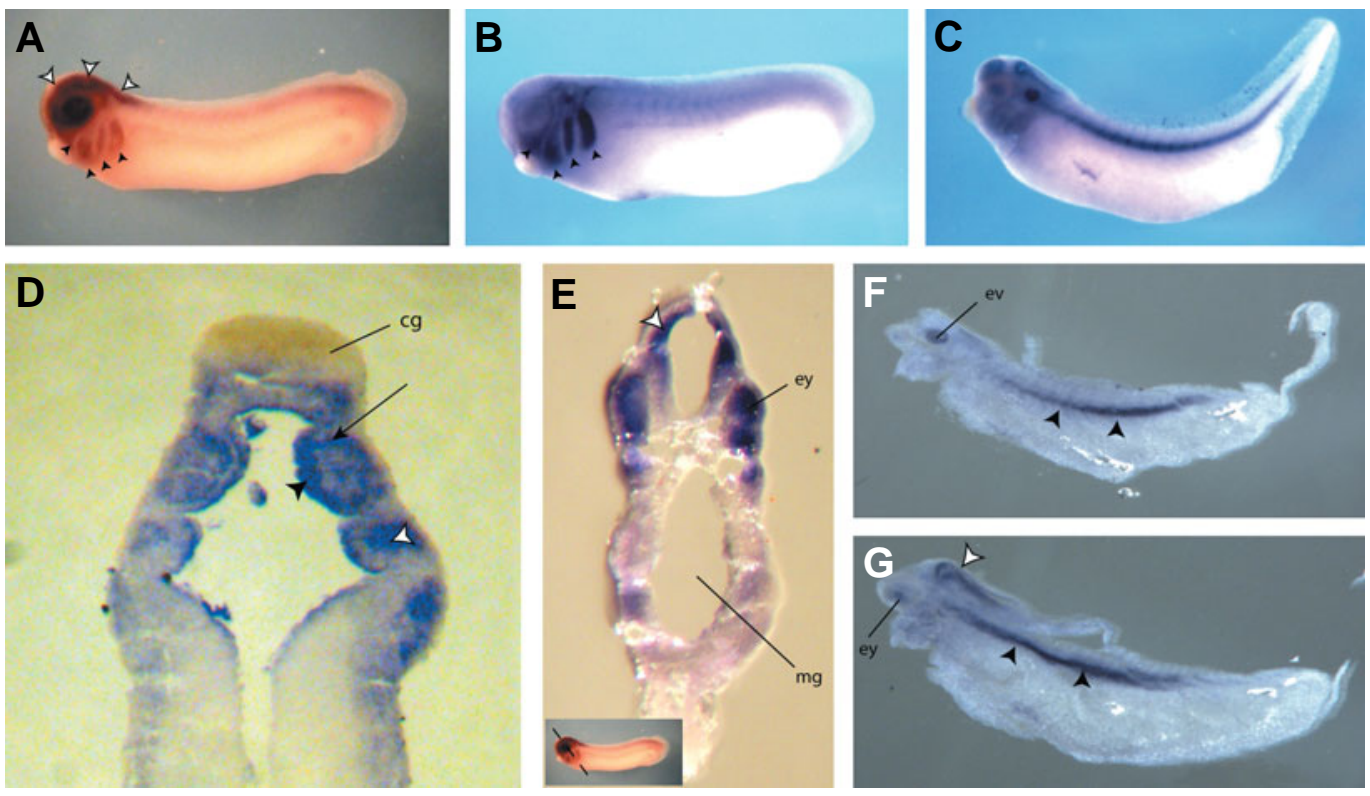

Fig. 3. Whole mount in situ hybridization of developing Xenopus embryos for Fmr1. (A) Early tailbud stage embryo (st. 26) showing Fmr1 expression in the CNS (white arrowheads) and pharyngeal arches (black arrowheads). (B) Expression of AP-2 $\alpha$, a neural crest marker at tailbud stage (st. 26). In comparison to Fmr1 expression pattern, AP-2 $\alpha$ expression is mostly limited to the pharyngeal arches at this stage (black arrowheads). (C) Fmr1 expression at early tadpole stage embryo (st. 26; see $F$ and $G$ for details). (D) Horizontal section of st. 26 embryo at the level of cement gland showing Fmr1 expression in endodermal (black arrowhead), mesodermal (black arrow) and mesenchymal (white arrowhead) regions of the pharyngealarches, with slight staining in the ectodermal region. The difference in staining intensity between the left and right side is due to the oblique nature of the sectioned tissue. Cement gland (cg) is negative for Fmr1 expression. (E) Oblique transverse section of tailbud stage embryo (st. 26). Strong expression is observed in the eye evagination (ey) and in CNS (white arrowhead). Insets show approximate region that has been sectioned (mg: midgut). (F,G) Sagittal section of late tailbud stage embryo depicted in Fig. $3 C$ showing strong expression in the CNS (G: white arrowhead), craniofacial regions, notochord (F, G: black arrowheads), ear vesicle (F: ev) and eye (G: ey).

In this paper, we demonstrated the developmental expression pattern of Fmr1 in Xenopus embryos. Although previous studies have hinted at Fmr1 expression in the neural crest cells and branchial arches in mice (Agulhon et al., 1999, de Diego Otero, 2000), our present data provide a clear evidence for Fmr1 expression in these regions. Because various tissue structures affected in FXS are derivatives of cranial neural crest, our findings carry important implications for understanding the FXS pathogenesis. Therefore, further examination of the role of Fmr1expression in the pharyngeal arches as well as various craniofacial regions would undoubtedly enhance our understanding of the Fragile $X$ pathology. Finally, since many tools are available to study various developmental processes using Xenopus development, this system should serve as an excellent animal model to investigate the role of $F m r 1$ expression during embryonic development.

\section{Experimental Procedures}

\section{Protein alignment and phylogenetic analysis}

The protein multiple sequence alignment was constructed with DS Gene 1.5 (Accelrys). Phylogenetic analysis was performed with DS Gene 1.5 using the Neighbor Joining Method and bootstrap analysis was performed with 1,000 resampled datasets.

\section{Northern blot hybridization}

RNAs were analyzed on Glyoxal gels (Ambion, Inc). Northern blots were washed in $0.2 \mathrm{XSSE}$ at $65^{\circ} \mathrm{C}$. Fmr1 probe was labeled with 32P-dCTP by primer extension (Amersham Pharmacia).

\section{In situ hybridization}

Whole-mount in situ hybridization was performed as described previously (Harland, 1991) using digoxygenin antisense probes for Xenopus Fmr1 and AP-2 $\alpha$ (Luo et al., 2003). Xenopus Fmr1 antisense probe was synthesized from pGEM72XFmr1 plasmid (Siomi et al., 1995) containing the full-length Xenopus Fmr1 using primers: forward 5'-cgg cga tct aga CTC CAA TGG AGC TTT CTA C-3'; reverse 5'-gca taa gga gct cTC AAT TGC AGT CAC CCC AG-3' (Xba1 and Sac1 sites are underlined in the primer sequence, respectively). The PCR product was digested with Xba1 and Sac1, gel purified and subcloned into Xba1 and Sac1 sites in pBluescript SK- vector (Stratagene). It was then linearized with $\mathrm{Xba} 1$ and in vitro transcribed in the presence of digoxygenin and T3 polymerase

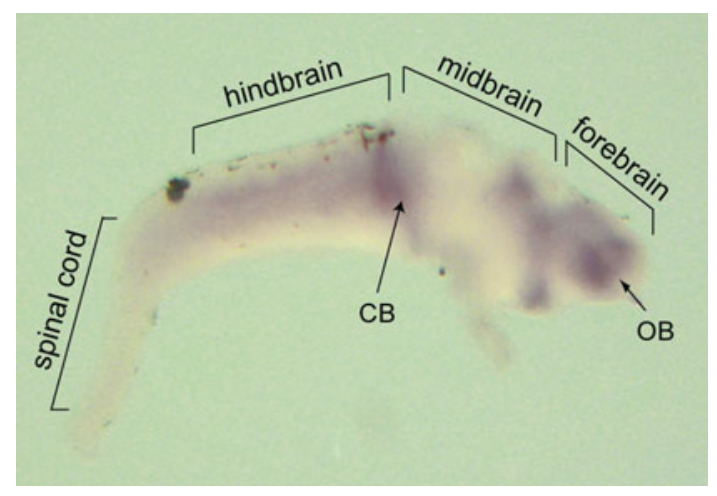

Fig. 4. Whole-mount in situ hybridization of Fmr1 in tadpole brain. The forebrain, midbrain and hindbrain (lateral view) show moderate to heavy Fmr1 expression. Abbreviations: ob, olfactory bulb; cb, cerebellum. 
(Roche Molecular).

\section{Acknowledgements}

We thank Dr. Gideon Dreyfuss for the pGEM72-Fmr1 plasmid and Dr. Kim Mowry and Dr. Tracy Kress for advice and access to their Xenopus facility. We also thank Dr. Yanhui Zhang for technical assistance. This work was supported by NIMH Predoctoral NRSA fellowship MH065094-02 (J.H.L) and by grants from National Institutes of Health (NS39321; RR15578). We also thank NICHD for their support.

\section{References}

AGULHON, C., BLANCHET, P., KOBETZ, A., MARCHANT, D., FAUCON, N., SARDA, P., MORAINE, C., SITTLER, A., BIANCALANA, V., MALAFOSSE, A. et al. (1999). Expression of fmr1, fxr1 and fxr2 genes in human prenatal tissues. J Neuropathol Exp Neuro/58: 867-80.

DARNELL, J.C., JENSEN, K.B., JIN, P., BROWN, V., WARREN, S.T. and DARNELL, R.B. (2001). Fragile $x$ mental retardation protein targets $g$ quartet mRNAs important for neuronal function. Cel/ 107: 489-99.

DE DIEGO OTERO, Y., BAKKER, CE. RAGHOE, P., SEVERIJNEN, L., HOOGEVEEN, A., OOSTRA, B.A., WILLEMSEN, R. (2000). Immunocytochemical characterization of fmrp, fxr1p and fxr2p during embryonic development in the mouse. Gene Funct. Dis. 1: 28-27.
HAGERMAN, R. and HAGERMAN, P. (2002). Fragile $x$ syndrome: Diagnosis, treatment and research. Johns Hopkins University Press, Baltimore.

HARLAND, R.M. (1991). In situ hybridization: An improved whole-mount method for xenopus embryos. Methods Cell Bio/36: 685-95.

HERGERSBERG, M., MATSUO, K., GASSMANN, M., SCHAFFNER, W., LUSCHER, B., RULICKE, T. and AGUZZI, A. (1995). Tissue-specific expression of a fmr1/beta-galactosidase fusion gene in transgenic mice. Hum Mol Genet 4: 359-66.

LUO, T., MATSUO-TAKASAKI, M., THOMAS, M.L., WEEKS, D.L. and SARGENT, T.D. (2002). Transcription factor AP-2 is an essential and direct regulator of epidermal development in xenopus. Dev Bio/245: 136-44.

LUO, T., LEE, Y.H., SAINT-JEANNET, J.P. and SARGENT, T.D. (2003). Induction of neural crest in xenopus by transcription factor AP-2alpha. Proc Nat/ Acad Sci USA 100: 532-7.

SIOMI, M.C., SIOMI, H., SAUER, W.H., SRINIVASAN, S., NUSSBAUM, R.L. and DREYFUSS, G. (1995). Fxr1, an autosomal homolog of the fragile $x$ mental retardation gene. EMBO J14: 2401-8.

Received: May 2005 Reviewed by Referees: June 2005 Modified by Authors and Accepted for Publication: July 2005 Edited by: George Malacinski 